\title{
Obtención del colorante natural del Camote (Ipomoea batatas)
}

\section{Natural dye extraction from Sweet Potato (Ipomoea batatas)}

\begin{abstract}
Raquel Leticia Coba Carrera ${ }^{1}$, Lady Gabriela Apolo Criollo ${ }^{2}$, José Homero Segura Mestanza ${ }^{3}$
\end{abstract} \& Hannibal Lorenzo Brito Moina ${ }^{4}$

Recibido: 28-04-2019 / Revisado: 15-05-2019 /Aceptado: 30-06-2019/ Publicado: 25-07-2019

\begin{abstract}
.
DOI: https://doi.org/10.33262/cienciadigital.v3i3.2.713

Artificial dyes are questioned for their harmful effects on the health of human beings, so it is intended to replace them with natural dyes that are healthy and their costs are low, for this reason the extraction of natural dyes from the sweet potato peel (Ipomoea batatas), for food use, for which, we proceeded with the proximal analysis to determine the moisture content, ashes, fiber and protein of the raw material, later in the soxhlet equipment the extraction was carried out using fresh and dry plant material that was obtained in the tray dryer and as solvent ethanol and citric acid, the extract was subjected to a separation process in a Rotavapor at a temperature of $50^{\circ} \mathrm{C}$ to obtain only the natural anthocyanin pigment, after this was done the physical-chemical, microbiological analysis.
\end{abstract}

Keywords: Food production, Coloring, Natural, Tuna, Drying, Lyophilization Extraction,

\section{Resumen.}

Los colorantes artificiales son cuestionados por sus efectos nocivos en la salud de los seres humanos, por lo que, se pretende reemplazar los mismos por colorantes naturales que son saludables y sus costos son bajos, por este motivo se realizó la extracción del

1 Escuela Superior Politécnica de Chimborazo, Facultad de Ciencias. Riobamba, Ecuador. raquel.coba@espoch.edu.ec

2 Escuela Superior Politécnica de Chimborazo, Facultad de Ciencias. Riobamba, Ecuador. gramy_lady@hotmail.com

3 Escuela Superior Politécnica de Chimborazo, Hospital de especialidades Abel Gilbert Pontón. Riobamba, Ecuador. johomer31s@gmail.com

4 Escuela Superior Politécnica de Chimborazo, Facultad de Ciencias. Riobamba, Ecuador. hbrito@espoch.edu.ec 
colorante naturales de la cáscara de camote (Ipomoea batatas), para uso alimenticio, para lo cual, se procedió con el análisis proximal para la determinación del contenido de humedad, cenizas, fibra y proteína de la materia prima, posteriormente en el equipo soxhlet se efectuó la extracción utilizando material vegetal fresco y seco que se obtuvo en el secador de bandejas y como solvente etanol y ácido cítrico, el extracto fue sometido a un proceso de separación en un Rotavapor a una temperatura de $50^{\circ} \mathrm{C}$ para obtener solo el pigmento natural antocianina, posterior a ello se realizó el análisis físico-químico, microbiológico.

Palabras claves: Antocianinas, Colorante, Natural, Tuna, Secado, Liofilización Extracción, Alimentos.

\section{Introducción.}

Los colorantes (Brito \& et al, Obtención y determinación de la calidad de colorante a partir de las flores de Sangorache, 2019) son compuestos químicos utilizados por su propiedad de tinción, resaltan el color original o proveen nuevas coloraciones en productos alimenticios, farmacéuticos, cosméticos y textiles, tienen como finalidad hacerlos más llamativos a la vista del consumidor, estos pueden ser naturales si provienen de fuentes vegetal, animal o mineral y artificiales si han sido modificados química o físicamente. (Sánchez, 2013; Yusuf, Shabbir, \& Mohammad, 2017).

Los colorantes naturales (Brito \& et al, Colorantes naturales para uso alimenticio, 2019) se tienen como grupos funcionales antocianinas (Ac), pigmentos hidrosolubles de coloración rojo, violeta y azul, se localiza en la composición química de frutos y vegetales rojos, productos agrícolas que se valorizan y se convierten en una excelente fuente de extracción de colorantes que se emplean en industrias alimenticias.

Los colorantes son comercializados por China, España, Francia, Italia y Turquía principalmente, la mayor producción está encabezada por los carotenoides con el 34\%, caramelo $32 \%$, antocianinas $24 \%$ y el resto en un $10 \%$, de esta manera la industria de los colorantes pretende para el 2020 dichas cifras hayan incrementado en un 6,4\% (Ministerio de Agricultura de Chile, 2017).

\section{Metodologia.}

Se procedió a determinar el contenido de humedad por el método gravimétrico a $105 \pm 3^{\circ} \mathrm{C}$, las cenizas en una mufla mediante la incineración a $505 \pm 3^{\circ} \mathrm{C}$, la fibra de acuerdo a la Norma 
Técnica Ecuatoriana (NTE INEN-542) y proteína en base a la Normativa de Asociación Oficial de Químicos Agrícolas (AOAC 984.13A). La materia vegetal se obtuvo mediante la asepsia donde se retiró impurezas, luego se peló con una solución de metabisulfito de sodio $\left(\mathrm{Na}_{2} \mathrm{~S}_{2} \mathrm{O}_{5}\right)$ al $1 \%(\mathrm{P} / \mathrm{V})$ para evitar su pardeamiento enzimático.

Se utilizó $50 \mathrm{~g}$ de material vegetal fresco y secado que se obtuvo en un secador de bandejas a una temperatura de $45^{\circ} \mathrm{C}$. A continuación, se procedió a extraer el colorante mediante el método de soxhlet, utilizando como solvente una solución acidificada de etanol 90\%-ácido cítrico $0,03 \%(\mathrm{P} / \mathrm{V})$, posteriormente el extracto fue concentrado en un rotavapor BÜCHI 461 WATER BATH a $50 \pm 5^{\circ} \mathrm{C}$, de esta manera se obtuvo solo el colorante natural.

A este componente se realizó el análisis sensorial, solubilidad en agua y en una sustancia apolar (aceite vegetal comestible), se efectuó el análisis físico-químico donde se determinó el pH en un pH-metro digital, la densidad se aplicó el método de picnómetro, y el porcentaje de grados Brix $\left({ }^{\circ} \mathrm{Bx}\right)$ e índice de refracción en un REFRACTÓMETRO DIGITAL, se realizó la lectura en el Espectrofotómetro UV-VISIBLE a una longitud de onda $(\lambda)$ de 400-700 nm y Espectrofotómetro a un número de onda: $3700-500 \mathrm{~cm}^{-1}$, también se evaluó su calidad microbiológica para determinar Esherichia coli, coliformes totales y fecales, mohos y levaduras, mediante siembra en placa.

\section{Resultados.}

Tabla 1 Análisis proximal de la materia prima

\begin{tabular}{|c|c|c|c|c|}
\hline \multirow{2}{*}{ No. } & \multirow{2}{*}{ Parámetro } & \multirow{2}{*}{ Unidad } & \multicolumn{2}{|c|}{ Camote morado } \\
\hline & & & $\mathbf{V}_{\mathbf{E}}$ & $\mathbf{V}_{\mathbf{T}}$ \\
\hline 1 & Humedad & $\%$ & $69.30^{\mathrm{a}} \pm 0.10^{\mathrm{b}}$ & 69.0 \\
\hline 2 & Cenizas & $\%$ & $1.02 \pm 0.00$ & 1 \\
\hline 3 & Fibra & $\%$ & $1.1 \pm 0.01$ & 1.1 \\
\hline 4 & Proteína & $\%$ & $1.11 \pm 0.01$ & 1.1 \\
\hline
\end{tabular}

$\left({ }^{\mathrm{a}}\right)=$ Promedio de repeticiones $\mathrm{n}=2,\left({ }^{\mathrm{b}}\right)=$ Desviación estándar de $\mathrm{n}=2,\left(\mathrm{~V}_{\mathrm{T}}\right)=$ Valor teórico,

$\left(\mathrm{V}_{\mathrm{E}}\right)=$ Valor experimental 
El camote morado al ser un tubérculo posee un alto contenido de humedad 69,30\%, bajo contenido de cenizas $1,02 \%$, fibra $1,1 \%$ y proteína $1,11 \%$, estos porcentajes se encuentran dentro del rango de referencia, garantizando su estabilidad y calidad. (Ecuador, Instituto Ecuatoriano de Nutrición, 1975; Fundación Universitaria Iberoamericana, 2017). Estos valores se hallan dentro de los rangos de referencia, de esta manera aportan para que la materia prima que va a ser utilizada en la extracción de colorantes naturales sea de calidad y presente condiciones óptimas. (Fundación Universitaria Iberoamericana, 2017; Perú, Ministerio de Salud, 2009).

Tabla 2 Extracción de colorantes del material vegetal fresco

\begin{tabular}{clc}
\hline No. & Parámetro & Camote morado \\
1 & Parte vegetal utilizada & Cáscara \\
2 & Cantidad $(\mathrm{g})$ & $50^{\mathrm{a}} \pm 0.00^{\mathrm{b}}$ \\
3 & Solvente $(\mathrm{mL})$ & $400 \pm 0.00$ \\
4 & Tiempo de extracción $(\mathrm{min})$ & $309 \pm 1.00$ \\
5 & Extracto $(\mathrm{mL})$ & $362 \pm 0.58$ \\
6 & Tiempo de concentración $(\mathrm{min})$ & $120 \pm 0.58$ \\
7 & Solvente recuperado $(\mathrm{mL})$ & $247 \pm 1.00$ \\
8 & Colorante $(\mathrm{mL})$ & $47 \pm 1.00$ \\
9 & Rendimiento $(\%)$ & $11.75 \pm 0.25$ \\
\hline
\end{tabular}

De la extracción de colorantes naturales a partir del material vegetal fresco (Tabla 2), la col morada por su textura más blanda fue el producto agrícola que requirió un menor tiempo para la extracción de su colorante natural $252 \pm 0.58$ min, además mediante la concentración en el rotavapor se pudo recuperar hasta $234 \pm 0.58 \mathrm{~mL}$ de solvente y se obtuvo $56 \pm 0.58 \mathrm{~mL}$ de pigmento natural con un mayor rendimiento en porcentaje de volumen $14 \pm 0.25 \%$.

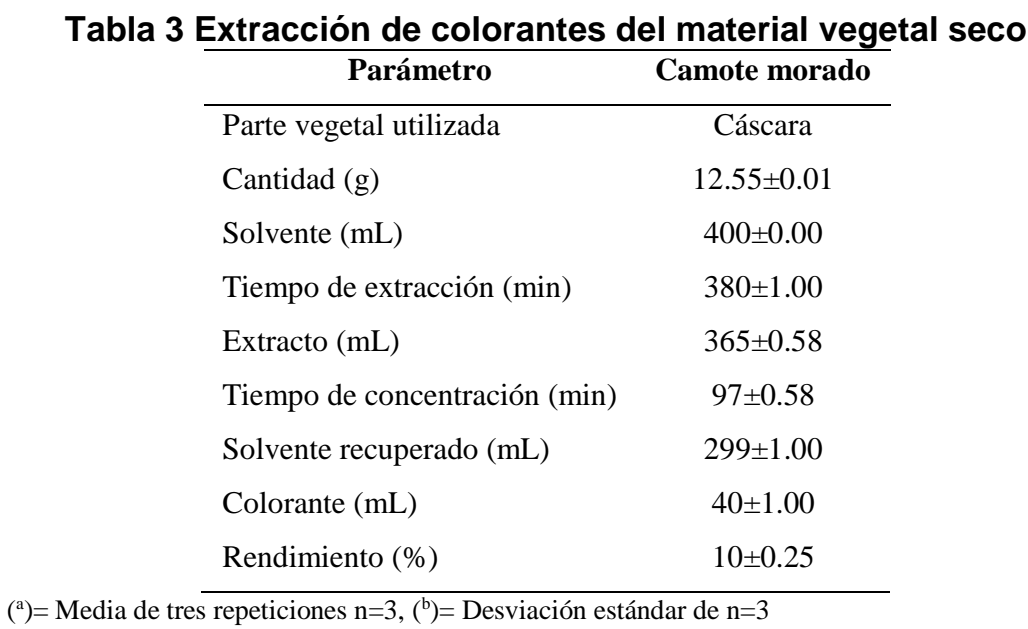


Para la extracción de colorantes naturales a partir de material vegetal seco (Tabla 3), se utilizó la cáscara de camote $12.55 \pm 0.01 \mathrm{~g}$, del cual se obtuvieron tras el secado de $50 \mathrm{~g}$ de material vegetal fresco en un secador de bandejas tipo armario a $45^{\circ} \mathrm{C}$. De donde el producto agrícola por su textura requirió un menor tiempo para la extracción de su colorante natural $356 \pm 0.58 \mathrm{~min}$, además mediante la concentración en el rotavapor se pudo recuperar hasta $305 \pm 0.58 \mathrm{~mL}$ de solvente y se obtuvo $49 \pm 1.00 \mathrm{~mL}$ de pigmento natural con un mayor rendimiento en porcentaje de volumen $12.25 \pm 0.25 \%$.

El material vegetal seco (Tabla 2) requirió un mayor tiempo para la extracción del colorante en comparación con el material vegetal fresco (Tabla 3), se asume que estas diferencias están relacionadas con el proceso de secado al cual fue sometido donde se ha reducido el contenido de humedad, por lo tanto, la muestra de material vegetal requiere un mayor tiempo para que el solvente penetre en él y arrastre los componentes.

Tabla 4 Análisis organoléptico y solubilidad de los colorantes naturales

\begin{tabular}{cccccc}
\hline \multirow{2}{*}{ Colorante } & \multirow{2}{*}{ Color } & \multirow{2}{*}{ Apariencia } & \multicolumn{2}{c}{ Solubilidad } \\
& & & & Agua destilada & Aceite vegetal \\
\hline \multirow{2}{*}{ Camote morado } & Fresco & Rojo & Líquida & Soluble & Insoluble \\
& Seco & Rojo intenso & Líquida & Soluble & Insoluble \\
\hline
\end{tabular}

El pigmento natural fue de color rojo para el camote fresco y rojo intenso para el seco, al comparar con otros estudios y la normativa mexicana para colorantes orgánicos, recalcan que las antocianinas abarcan colores como el rojo, violeta y azul, lo que permite aplicar en los alimentos como colorantes naturales. (México-NOM-119-SSA1, 1994; Carocho, Morales, \& Ferreira, 2015).

En lo que respecta a la solubilidad se determinó que los colorantes naturales fueron solubles en agua e insolubles en aceite vegetal comestible donde se evidenció sinéresis, según otros estudios indican que las antocianinas son pigmentos visibles al ojo humano, solubles en agua con una posible aplicación de colorantes en diferentes productos alimenticios. (Mizgier et al., 2016; Aguilera, Reza, Chew, \& Meza, 2011). 
Tabla 5 Resultados del análisis físico-químico

\begin{tabular}{clcccc}
\hline \multicolumn{2}{c}{ Colorante } & $\mathbf{p H}$ & $\boldsymbol{\rho}(\mathbf{g} / \mathbf{m L})$ & ${ }^{\circ} \mathbf{B x}(\%)$ & $\mathbf{n D}$ \\
\hline \multirow{2}{*}{ Camote morado } & Fresco & $4.48^{\mathrm{a}} \pm 0.04^{\mathrm{b}}$ & $0.9314 \pm 0.02$ & $16.23 \pm 1.65$ & $1.3711 \pm 0.01$ \\
& Seco & $4.46 \pm 0.04$ & $0.9704 \pm 0.02$ & $26.29 \pm 1.68$ & $1.3758 \pm 0.00$ \\
\hline
\end{tabular}

$(\rho)=$ Densidad relativa, $\left({ }^{\circ} \mathrm{Bx}\right)=$ Grados brix,$(\mathrm{nD})=$ Índice de refracción,

$\left({ }^{a}\right)=$ Media de tres repeticiones $n=3,\left({ }^{b}\right)=$ Desviación estándar de $n=3$

Los pigmentos naturales extraídos posee $\mathrm{pH}$ ácidos (Tabla 5), esto favorece su estabilidad puesto que las antocianinas están presentes como cationes flavilo (Kamiloglu et al., 2015). La densidad de los colorantes naturales es inferior al del agua $1 \mathrm{~g} / \mathrm{mL}$ y superior a la del etanol $0,789 \mathrm{~g} / \mathrm{mL}$, se asume que esto se debe a un alto contenido de sustancias solubles o compuestos fitoquímicos. En lo que se refiere a los grados Brix $\left({ }^{\circ} \mathrm{Bx}\right)$ e índice de refracción (nD) se destaca el pigmento natural de camote morado con valores en fresco de $16,23{ }^{\circ} \mathrm{Bx}$, $1,3711 \mathrm{nD}$ y en seco $26,29{ }^{\circ} \mathrm{Bx}, 1,3758 \mathrm{nD}$, esto se relaciona a que dentro de su composición química el camote posee almidón el cual al convertirse en azúcares incrementa sus valores en la solución.

Tabla 6 Concentración total de antocianinas en los colorantes

\begin{tabular}{llrr}
\hline \multirow{2}{*}{ Parámetro } & \multirow{2}{*}{ Unidad } & \multicolumn{2}{c}{ Camote morado } \\
& & Fresco & Seco \\
\hline Longitud de onda $(\lambda)$ & $\mathrm{Nm}$ & 530 & 518 \\
Concentración total de & $\mathrm{mg} / \mathrm{L}$ & 67.96 & 179.18 \\
antocianinas & & & \\
Factor de dilución & - & 10 & 10 \\
\hline
\end{tabular}

Mediante la lectura en el espectrofotómetro (Tabla 6) se determinó la concentración total de antocianinas sin embargo no se puede especificar el tipo al que corresponde puesto que no se dispone de los estándares para dicha cuantificación.

\section{Conclusiones.}

- La temperatura de secado es de $45 \mathrm{C}$.

- La extracción del colorante se la hace a una temperatura de $50 \mathrm{C}$.

- La densidad de los colorantes naturales es inferior al del agua $1 \mathrm{~g} / \mathrm{mL}$ y superior a la del etanol $0,789 \mathrm{~g} / \mathrm{mL}$.

- Los grados Brix $\left({ }^{\circ} \mathrm{Bx}\right) 16,23{ }^{\circ} \mathrm{Bx}$ e índice de refracción 1,3711 (nD). 
- Los valores obtenidos en los ensayos de colorante cumplen con la norma internacional.

\section{Referencias bibliográficas.}

Ames, B., Shigena , M., \& Hagen , T. (2000). Oxidants, antioxidants and the degenarative diseases of aging.

Amold , L., Lofthouse , N., \& Hurt, E. (2002). Artifcial food colors and attentiondefcit/hyperactivity symptom conclusions to dye for. Neurotherapeutics ,.

Ansorena , M., Moreira , M., \& Roura, S. (2014). Combined effect of ultrasound, mild heat shock and citric acid to retain greenness, nutritional and microbiological quality of minimally processed broccoli (Brassica oleracea L.): an optimization study.

Babu , B., Rastogi , N., \& Raghavarao, K. (2008). Concentration and temperature polarization effectts during osmotic membrane distillation. Journal of membrane sciencia.

Badui , A., \& Degal , S. (2006). Química de los alimentos. México: Pearson.

Bavec, M., \& et al. (2010). Influence of industrial and alternative farming systems on contents of sugars, organic acids, total phenolic content, and the antioxidant activity of red beet (Betavulgaris L. ssp. vulgaris Rote Kugel). Journal of Agricultural and Food Chemistry.

Beers, E., Woffenden , B., \& Zhao , C. (2000). Plant proteolytic enzymes: possible roles during programmed cell death. Plant Mol Biol 2000.

Bell , \& Wagstaff, C. (2014). Glucosinolates, Myrosinase Hydrolysis Products, and Flavonols Found in Rocket (Eruca sativa and Diplotaxis tenuifolia).

Bergquist , S., \& Gertsson. (2017). Ascorbic acid,carotenoids, and visual quality of baby spinach as affected by shade netting and postharvest storage. Journal of Agricultural and Food Chemistry. Journal of Agricultural and Food Chemistry.

Bergquist, S., \& et al. (2007). Ascorbic acid, carotenoids and visual quality of baby spinach as affected by shade netting and postharvest. Journal of Agricultural and Food Chemestry. 
Borja, D., Chango, G., Vacacela, P., \& Brito, H. (2019). Obtaining Yacon Flour (Smallanthus sonchifolius). International Journal of Current Research and Academic Review.

Brito, H. (2000). Texto Básico de Operaciones Unitarias I. Riobamba, Chimborazo, Ecuador: Docucentro ESPOCH.

Brito, H. (2001). Texto Básico de Operaciones Unitarias III. Riobamba: Docucentro ESPOCH.

Brito, H. (2006). Texto Básico de Mecánica de Fluidos. Riobamba.

BRITO, H., \& et al. (2016). DISEÑO Y CONSTRUCCIÓN DE UN LIOFILIZADOR PARA EL SECADO DE LA REMOLACHA AZUCARERA (Beta vulgaris var. saccharifera. Riobamba, Chimborazo, Ecuador.

Brito, H., \& et al. (2016). Obtención de alcohol a partir de remolacha. European Journal of Scientific Research.

Brito, H., \& et al. (septiembre de 2017). Diseño de un proceso industrial para la obtención de bioetanol a partir de lactosuero. Riobamba.

Brito, H., \& et al. (2017). Diseño y construcción de un clarificador para la bebida del Tzawar Mishki en la producción de alcohol. Riobamba.

Brito, H., \& et al. (2019). Colorantes naturales para uso alimenticio. Ciencia Digital. doi:10.33262/cienciadigital.v3i2.4.510

Brito, H., \& et al. (2019). Obtención y determinación de la calidad de colorante a partir de las flores de Sangorache. Ciencia Digital.

Bui, A, V., Ngyen, M, H., \& Joachim, M. (2004). laboratory study on glucose concentration by osmotic distillation in hollow fibre module. JOURNAL OF FOOD ENGINEERING, 237-245.

Calvo, I. (2009). EL CULTIVO DE LA UCHUVA (Physalis peruviana). Microcuenca Plantón - Pacayas(10), 1-112.

Delgado-Vargas, F., Jimenez, A., Paredes-Lopez, O., Stintzing, \& Carle. (2004). Natural pigments:. Critical Reviews in Food Science and Nutrition, 173-289. Obtenido de https://www.clinicalkey.es/service/content/pdf/watermarked/1-s2.0S0140673607613063.pdf?locale=es_ES

Fischer, G., \& Miranda, D. (2012). Uchuva (Physalis peruviana L.). En Manual para el cultivo de frutales en el trópico (págs. 851-873). Bogotá: FISCHE R, G. (Ed.). 
Luque de Castro, M., \& Priego Capote, F. (2010). Soxhlet extraction: Past and present panacea. Journal of Chromatography A.

Mccann, D., Barrett, A., Cooper, A., Crumpler, D., Dalen, L., \& Grimshaw, K. (2007). A Randomised, Double-Blinded, Placebo-controlled Trial. Food additives and hyperactive behaviour in 3-year-old and, 1560-1567. Obtenido de https://www.clinicalkey.es/service/content/pdf/watermarked/1-s2.0S0140673607613063.pdf?locale=es_ES

SHAPIRO, A., \& et al. (2001). Chemoprotective Glucosinolates and Isothiocyanates of Broccoli Sprouts: Metabolism and Excretion in Humans. Cancer Epidemiology, Biomarkers \& Prevention.

Trujillo, C. J., \& Tapia, R. L. (2012). DETERMINACIÓN DE ADULTERACIÓN EN MAÍZ MORADO QUE SE EXPENDE EN MERCADOS DE LA CIUDAD. TRUJILLOJULIO. 


\section{PARA CITAR EL ARTÍCULO INDEXADO.}

Ureña Moreno, J. E., Renato Chávez, C., \& Parra León, V. (2019). La estera andina como aislante para la rehabilitación energética de viviendas rurales en el páramo ecuatoriano. Ciencia Digital, 3(3.2), 24-37. https://doi.org/10.33262/cienciadigital.v3i3.2.713

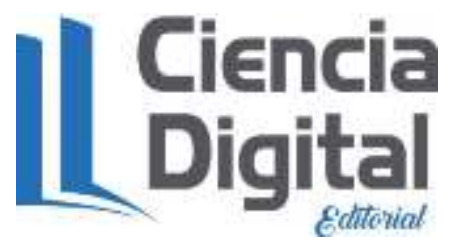

El artículo que se publica es de exclusiva responsabilidad de los autores y no necesariamente reflejan el pensamiento de la Revista Ciencia Digital.

El artículo queda en propiedad de la revista y, por tanto, su publicación parcial y/o total en otro medio tiene que ser autorizado por el director de la Revista Ciencia Digital.
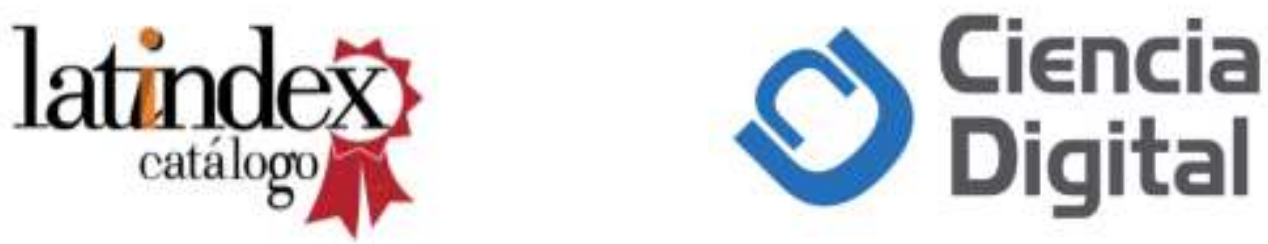